\title{
Fonte de alimentação trifásica, saída 60v e 100a, com fator de potência unitário e baixo nível de RFI
}

\section{Three-phase power supply, output 60v and 100a, with unit power factor and low RFI level}

\author{
Carlos Henrique Gonçalves Treviso'; Adriano Alves Pereira ${ }^{2}$; \\ Lúcio dos Reis Barbosa ${ }^{3}$; Luiz Carlos de Freitas ${ }^{4}$; João Batista Vieira Júnior ${ }^{5}$
}

\section{Resumo}

\begin{abstract}
Este artigo apresenta o funcionamento de um retificador trifásico, fator de potência unitário, comutação não dissipativa na conversão CC-CC e controle sincronizado em freqüência, funcionando com $89 \%$ de rendimento global com baixo nível de RFI. A estrutura proposta permite o funcionamento com alta freqüência de chaveamento. A tensão de saída é controlada por PWM com uma freqüência constante. O princípio de funcionamento, análise teórica da conversão CC, equações relevantes, técnica de controle para o balanceamento de corrente, resultados experimentais são mostrados neste trabalho.
\end{abstract}

Palavras Chave: Retificador, fator de potência, alto rendimento, baixo RFI

\begin{abstract}
This paper presents a three-phase rectifier, unit power factor, non-dissipative commutation in DC-DC converter and synchronized control in frequency, working with $89 \%$ of global efficiency and low level do RFI. The proposed structure allows the operation with high switching frequency. The output voltage is controlled by PWM with a constant frequency. The operation principle, theoretical analysis from DC converter, relevant equations, current balance control technique and experimental results, are shown in this paper.
\end{abstract}

Key Words: Rectifier, unit power, high performance, low RFI

\section{Introdução}

No Brasil, poucos setores estão preocupados realmente com RFI. Somente as indústrias nacionais que produzem equipamentos para os exigentes mercados externos e para as empresas de telecomunicações são obrigadas a realizarem testes de RFI em seus equipamentos (ASSOCIAÇÃO BRASILEIRA DE NORMAS TÉCNICAS, 1991; IEC-CISPR, 1990).

\footnotetext{
${ }^{1}$ Docente do Departamento de Engenharia Elétrica da Universidade Estadual de Londrina - e-mail: treviso@uel.br.

${ }^{2}$ Docente do Departamento de Engenharia Elétrica da Universidade Federal de Uberlândia - e-mail: adriano@eletrica.ufu.br

${ }^{3}$ Docente do Departamento de Engenharia Elétrica da Universidade Estadual de Londrina - e-mail: 1rbarbosa@uel.br.

${ }^{4}$ Docente do Departamento de Engenharia Elétrica da Universidade Federal de Uberlândia - e-mail: freitas@eletrica.ufu.br

${ }^{5}$ Docente do Departamento de Engenharia Elétrica da Universidade Federal de Uberlândia - e-mail: batista@ufu.br
} 
Por meio de contatos com fabricantes de renome na produção de retificadores, concluiu-se que a construção de um retificador, capaz de fornecer uma corrente de saída de 100A e uma tensão de $60 \mathrm{~V}$ em corrente contínua, além das características expostas no resumo e tomando-se especial cuidado com os ruídos de RFI, com acabamento semi-industrial, seria uma valiosa contribuição técnico-científica para o mercado nacional (TREVISO, 1999).

No sentido de ilustrar os objetivos deste trabalho, a Figura 1 traz o diagrama de blocos do retificador proposto.

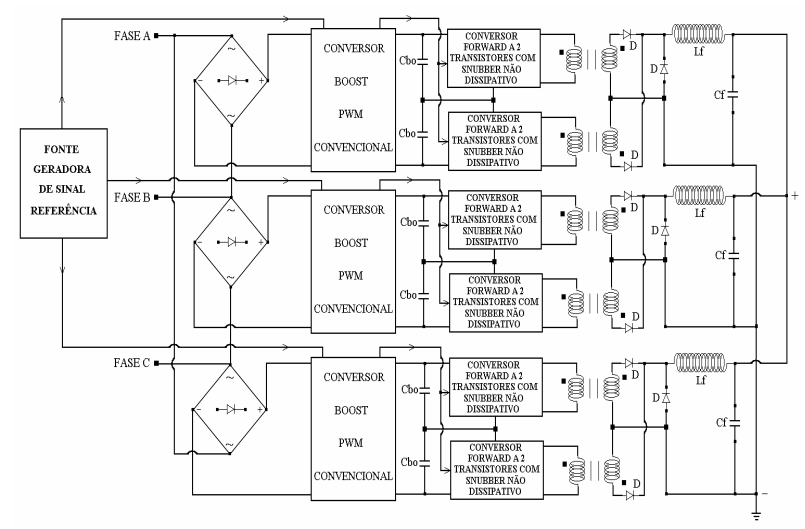

Figura 1 - Diagrama de Blocos do Retificador Trifásico.

Para o estágio CA-CC, utiliza-se o conversor Boost PWM convencional como pré-regulador, muito conhecido na literatura especializada e para a conversão CC-CC, utiliza-se o conversor Forward a 2 transistores com "snubber" não dissipativo multi-nível.

O casamento das freqüências dos módulos coloca os ruídos provenientes das derivadas de correntes e/ ou tensões em um espectro bem definido, tornandose assim, mais fácil a atenuação, constituindo-se em uma importante contribuição (CRUZ, 1996).

\section{Circuito de Geração de Sinais}

Neste circuito são implementados os sinais referências necessários para o casamento das freqüências de todos os conversores do retificador trifásico.
A Figura 2 mostra o esquema elétrico do circuito.

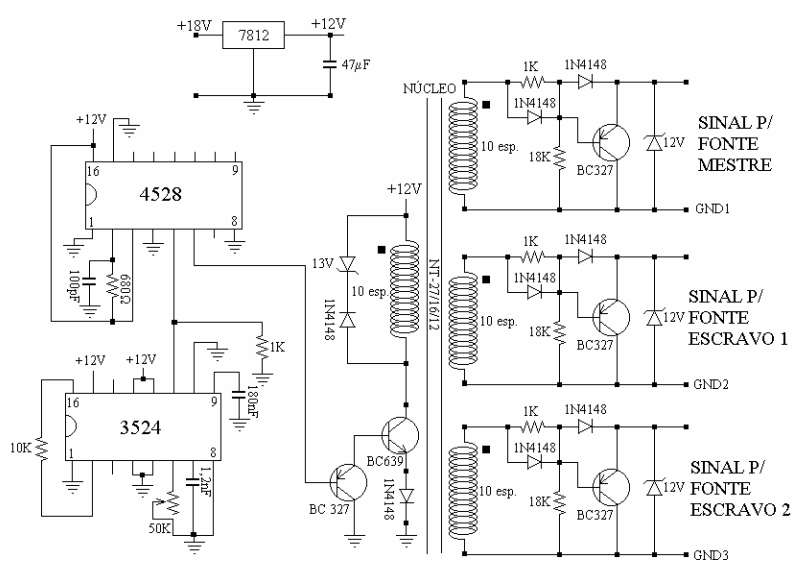

Figura 2 - Esquema Elétrico do Circuito de Geração de Sinais.

Este circuito é colocado em um conjunto CA-CC e CC-CC e será denominado módulo mestre e os demais conjuntos serão denominados módulos escravos.

\section{O Conversor Boost como Pré-Regulador}

O conversor Boost, com a devida técnica de controle, possibilita obter-se uma corrente de entrada senoidal e em fase com a tensão de entrada, além de um controle da tensão do barramento CC (UNITRODE, 1999).

Para a obtenção do exposto acima, utilizou-se o CI3854 para o controle do pré-regulador, cujo diagrama de blocos é mostrado na Figura 3.

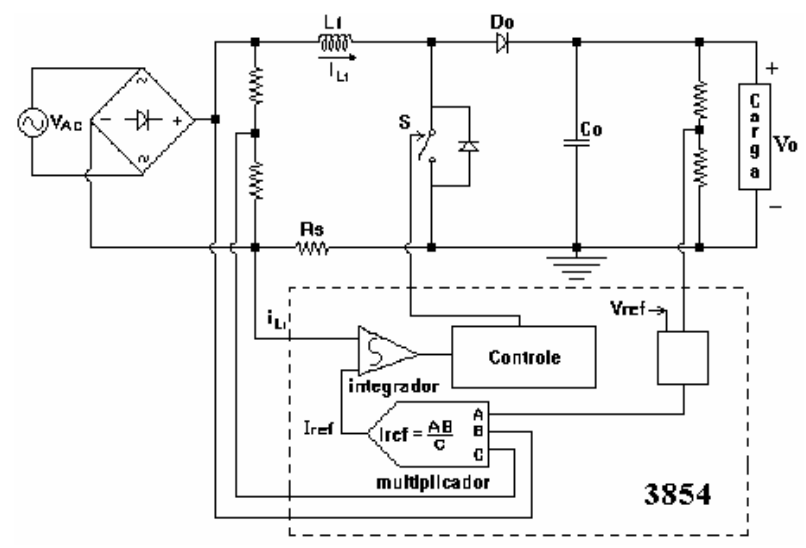

Figura 3 - Diagrama de Blocos do Controle Aplicado ao Conversor Boost. 
A técnica utilizada pelo CI3854 é o método de controle da corrente média.

O esquema elétrico completo com todos os componentes dimensionados para a potência de 2200 Watts, tensão de saída de $380 \mathrm{~V}$ e tensão de entrada variando de 185 a 250 VCA, é mostrado na Figura 4.

$\mathrm{Na}$ Figura 4, o sinal referência proveniente do circuito gerador de sinais faz o transistor BC337 descarregar o capacitor conectado ao pino 14 (3854), casando-se assim a freqüência e o "trimpot" de $12 \mathrm{~K}$ no pino 12 (3854), ajusta a amplitude da triangular. A partir da triangular, faz-se o isolamento do sinal que será utilizado casar a frequiência do circuito de controle do conversor CC-CC.

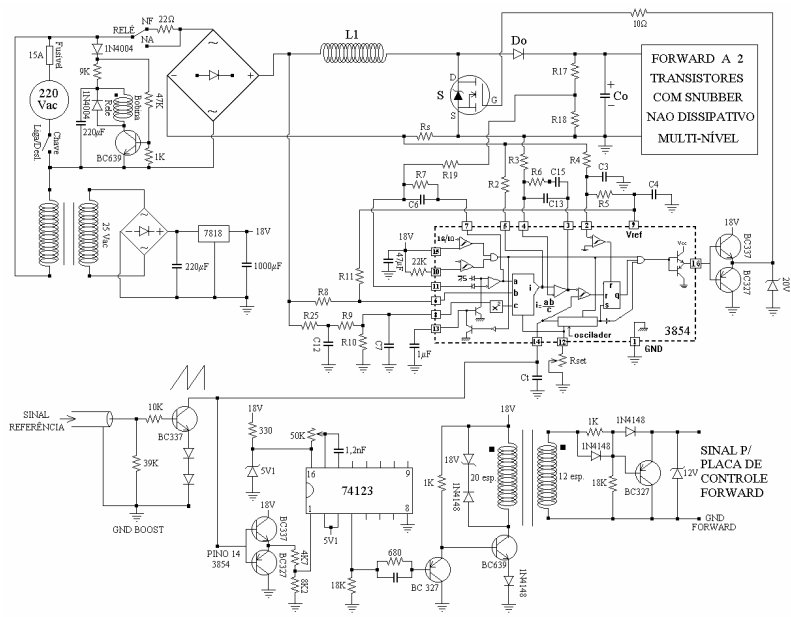

Figura 4 - Esquema Elétrico Completo do Pré-Regulador.

O circuito existente antes da ponte retificadora, faz a limitação da corrente de "inrush", cujo tempo para "curto-circuitar" a resistência de $22 \Omega ð$ (definido pelo resistor de $9 \mathrm{~K}$ e o capacitor de $220 \mu \mathrm{F}$ ) é $2 \mathrm{~s}$ aproximadamente.

\section{A Conversão CC-CC}

Para a conversão CC-CC, é utilizado o conversor Forward a 2 transistores com "snubber" não dissipativo multi-nível, que consiste em dois conversores Forward a 2 transistores com "snubber" não dissipativo no lado primário e os secundários acoplados. A Figura 5 mostra o circuito simplificado.

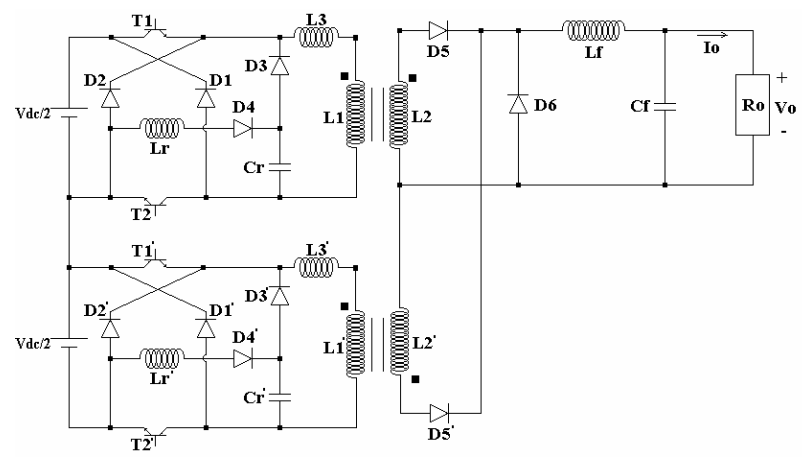

Figura 5 - Circuito Elétrico Simplificado Para a Conversão CC-CC.

As principais vantagens da topologia da Figura 5 são: as reduções das tensão nas chaves em $50 \%$ da tensão do barramento (saída do conversor Boost) e o indutor de saída é projetado para o dobro da freqüência de chaveamento de cada conversor Forward. O conversor Boost funciona numa frequiência de $100 \mathrm{KHz}$, enquanto que cada conversor do lado primário funciona a $50 \mathrm{KHz}$, porém defasados de 180 graus.

Para ilustrar o funcionamento da comutação não dissipativa do conversor Forward a 2 transistores, será feita a análise da estrutura da Figura 6.

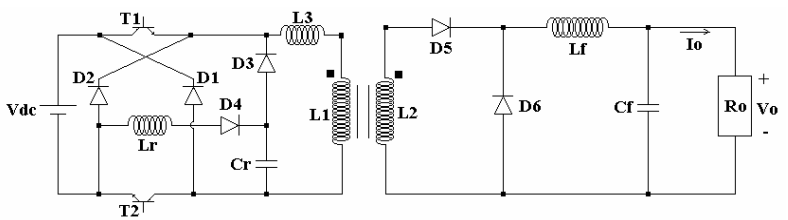

Figura 6 - Circuito simplificado do conversor Forward a 2 Transistor com Snubber não Dissipativo.

Os elementos do "snubber" não dissipativo da Figura 6 são: Lr, D3, D4, Cr e L3.

As vantagens desta topologia são: reduzido número de componentes e ausência de um transistor auxiliar para obter a ressonância. Para um projeto bem dimensionado, pode-se obter as mesmas características do conversor Forward a 2 transistores convencional do ponto de vista do controle (TREVISO, et al., 1998). 
A topologia da Figura 6 foi analisada de acordo com as seguintes considerações:

- Todos os semicondutores são ideais;

- A tensão de entrada é constante;

- A indutância magnetizante é muito grande;

- Não existe indutância de dispersão;

- A Corrente de saída é constante;

$-\mathrm{N} 1=\mathrm{N} 2$.

Este conversor possui 6 etapas de funcionamento para um ciclo de chaveamento.

Primeira etapa, $\left(\mathbf{t}_{\mathbf{0}}, \mathbf{t}_{\mathbf{1}}\right)$ - Etapa linear de corrente no indutor série L3. Inicia-se quando os transistores T1 e T2 entram em condução simultaneamente com correntes nulas (ZCS), devido a indutância L3. Neste estágio começa a ressonância entre $\mathrm{Lr}$ e $\mathrm{Cr}$, com a tensão no capacitor $\mathrm{Cr}$ variando de $-\mathrm{Vdc}$ até um valor $\mathrm{VCr} 1$, enquanto a corrente no indutor Lr varia de zero a um valor iLr1, terminando esta etapa quando ocorre o bloqueio do diodo de roda livre D6.

Segunda etapa, $\left(\mathbf{t}_{1}, \mathbf{t}_{2}\right)$ - Este estágio é a etapa PWM. Continua ocorrendo a ressonância entre Lre $\mathrm{Cr}$, com a tensão em Cr variando de $\mathrm{VCr} 1 \mathrm{a}+\mathrm{Vdc} \mathrm{e}$ a corrente iLr1 atinge um máximo e decresce até zero. Esta etapa termina quando os transistores $\mathrm{T} 1$ e T2 entram em corte.

Terceira etapa, $\left(\mathbf{t}_{2}, \mathbf{t}_{3}\right)$ - Nesta etapa ocorre o descarregamento linear do capacitor de ressonância. Inicia-se com a abertura dos transistores $\mathrm{T} 1$ e T2 sob tensões nulas (ZVS) devido ao capacitor de ressonância estar carregado com a tensão de entrada Vdc. A corrente de saída constante impõe uma descarga linear do capacitor $\mathrm{Cr}$, chegando à tensão nula quando termina esta etapa.

Quarta etapa, $\left(\mathbf{t}_{3}, \mathbf{t}_{4}\right)$ - Nesta etapa ocorre a ressonância entre o capacitor $\mathrm{Cr}$ e a indutância magnetizante do transformador. Este estágio iniciase quando a tensão no capacitor torna-se nula, polarizando diretamente o diodo de roda livre D6, devido a corrente de saída Io. A energia do transformador é transferida para o capacitor $\mathrm{Cr}$, invertendo sua polaridade, terminando esta etapa quando a tensão em $\mathrm{Cr}$ atingir-Vdc.

Quinta etapa, $\left(\mathbf{t}_{4}, \mathbf{t}_{5}\right)$ - Nesta etapa tem-se a desmagnetização do transformador. O início ocorre quando a tensão do capacitor atinge $-\mathrm{Vdc}$, polarizando os diodos D1 e D2. A corrente magnetizante circula através dos diodos, devolvendo energia para a fonte de entrada, terminando este estágio quando a corrente magnetizante torna-se nula.

Sexta etapa, $\left(\mathbf{t}_{5}, \mathbf{t}_{\mathbf{6}}\right)$ - Nesta etapa ocorre o grampeamento da tensão do capacitor $\mathrm{Cr}$ em -Vdc. Inicia-se quando a corrente magnetizante torna-se nula, permanecendo neste estado até ocorrer os disparos dos transistores. Quando termina este estágio, fecha-se o ciclo de chaveamento.

A Figura 7 mostra os circuitos equivalentes para cada etapa de funcionamento.
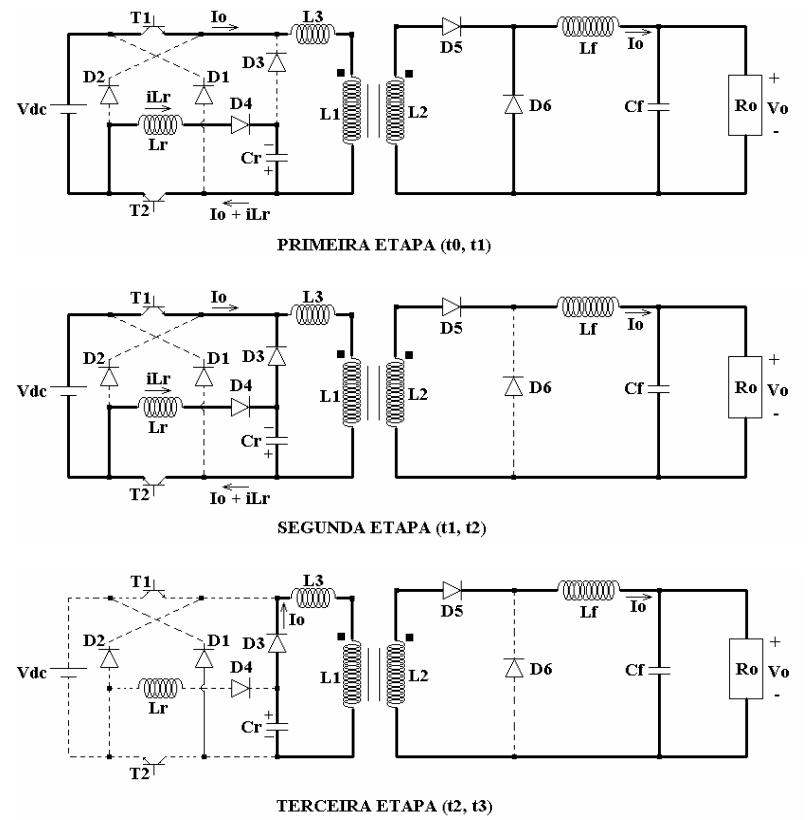

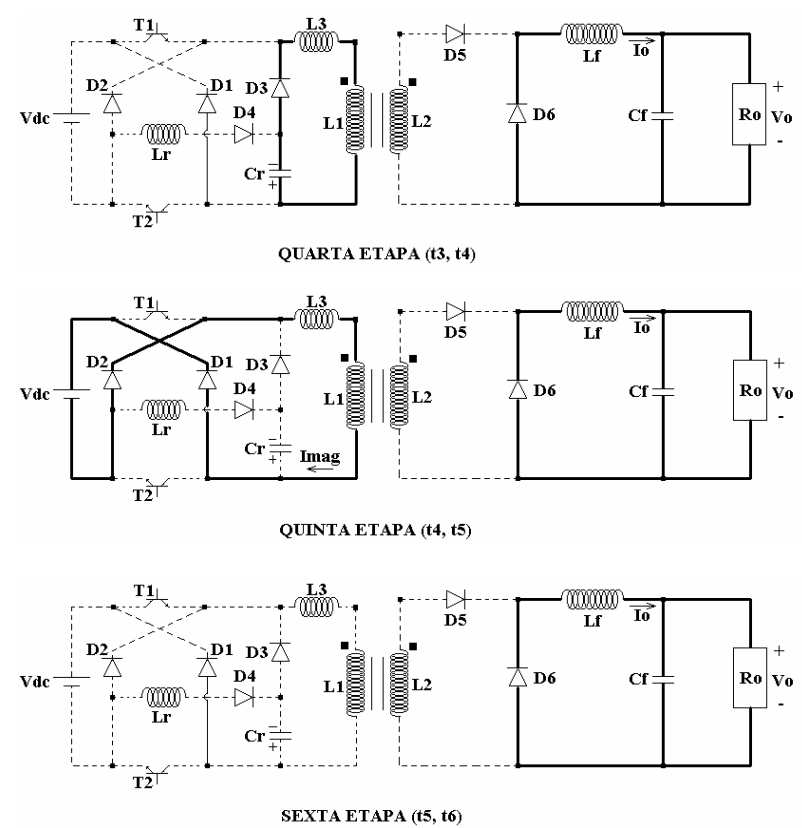

Figura 7 - Circuitos Equivalentes para Cada Etapa de Funcionamento Para Um Ciclo de Chaveamento.

As etapas de funcionamento deram origem às formas de onda mostrada na Figura 8.

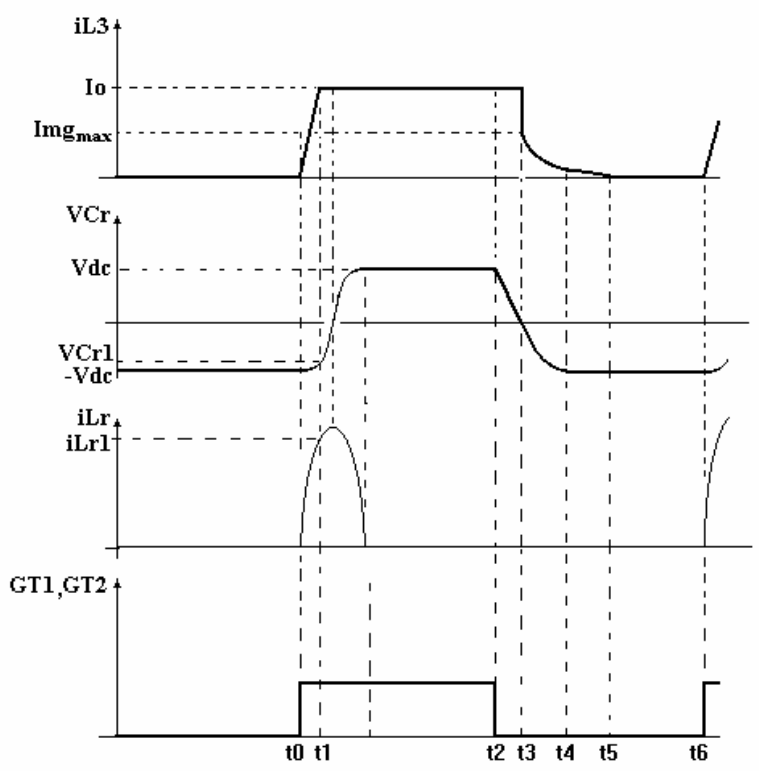

Figura 8 - Principais Formas de Onda Para os Circuitos Equivalentes.

A tensão de saída Vo, pode ser obtida por meio da análise dos estágios de funcionamento com as considerações expostas anteriormente para análise.
Partindo das considerações acima, chega-se à equação 1:

$$
\frac{V o}{V d c}=d-F s \bullet \frac{\alpha}{\omega o}+\frac{F s}{2 \bullet \omega o \bullet \alpha}
$$

onde:

$F s=$ freqüência de chaveamento;

$F o=$ frequiência de ressonância;

$$
\begin{gathered}
\omega o=2 \pi F o=\frac{1}{\sqrt{L_{R} C_{R}}} \\
\alpha=\frac{\left(I_{0}\right)}{V d c} \sqrt{\frac{L_{R}}{C_{R}}}
\end{gathered}
$$

A Figura 9 mostra a faixa de conversão $g$ para as equações acima.

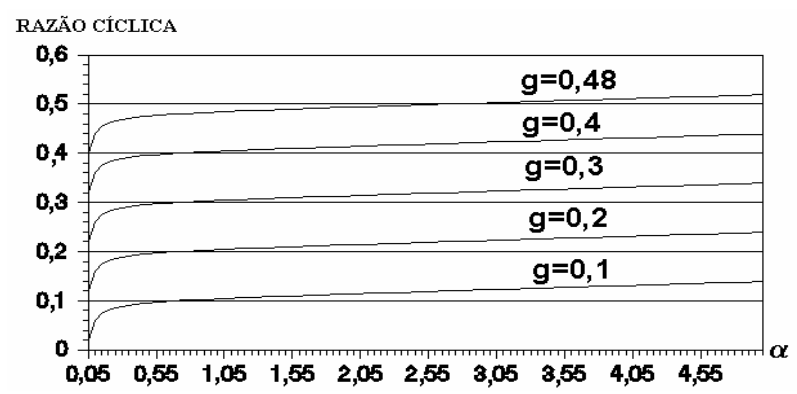

Figura 9 - Faixa de conversão para o conversor.

De acordo com a Figura 9, este conversor apresenta uma diminuição do ganho estático em relação a razão cíclica para a

\section{A Estratégia de Controle para a Conversão CC-CC}

Para realizar do controle da conversão CC-CC de cada módulo, será utilizado o CI3525.

O sincronismo da frequiência de chaveamento do conversor CC-CC com a frequiência do conversor 
Boost é realizado através do circuito elétrico mostrado na Figura 10.

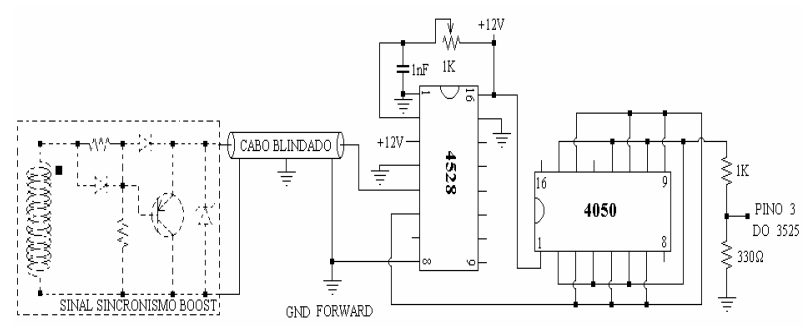

Figura 10 - Circuito Para Sincronizar Com Freqüência do Conversor Boost.

O pino 3 do CI3525 é a entrada para se fazer o sincronismo. O sinal proveniente do conversor Boost (Figura 4) possui isolação galvânica e entra no monoestável, produzindo pulsos de $2,8 \mathrm{~V}$ e largura de $0,5 \mathrm{~ms}$, realizando assim o casamento de frequiência.

A Figura 11 mostra o diagrama de blocos para a realização do controle do módulo mestre.

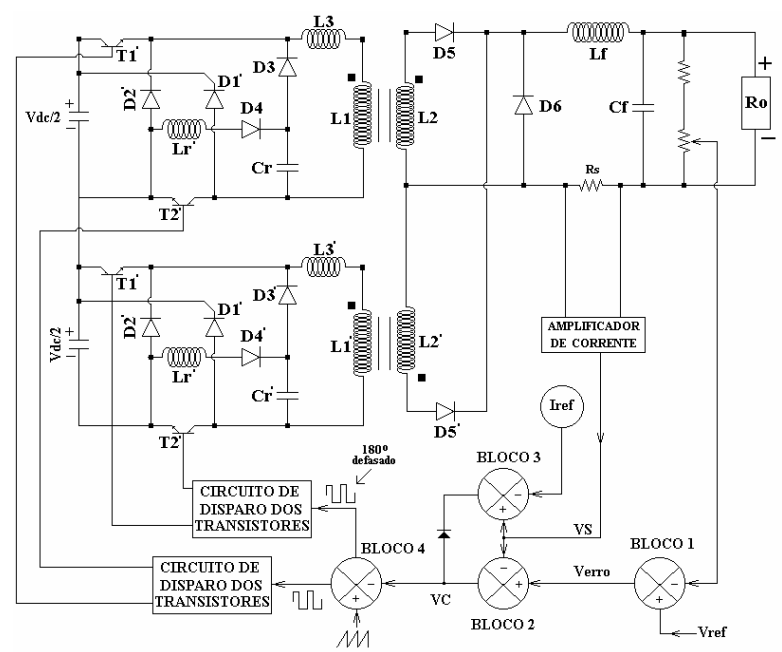

Figura 11 - O diagrama de blocos da estratégia de controle.

A tensão de saída, mostrada e comparada com uma tensão referência produz um sinal Verro (bloco 1). O amplificador de corrente produz um sinal de tensão Vs proporcional à corrente de saída. O sinal VS é comparado com os sinais Verro (bloco 2) e Iref (bloco 3). O sinal Vc pode ser gerado a partir dos blocos 2 ou 3 dependendo do valor de Iref e comparado com uma dente de serra (bloco 4), produzindo os pulsos para as chaves. O bloco circuito de disparo dos transistores faz a isolação galvânica dos pulsos.

A Figura 12 mostra o diagrama de blocos para a fonte trifásica.

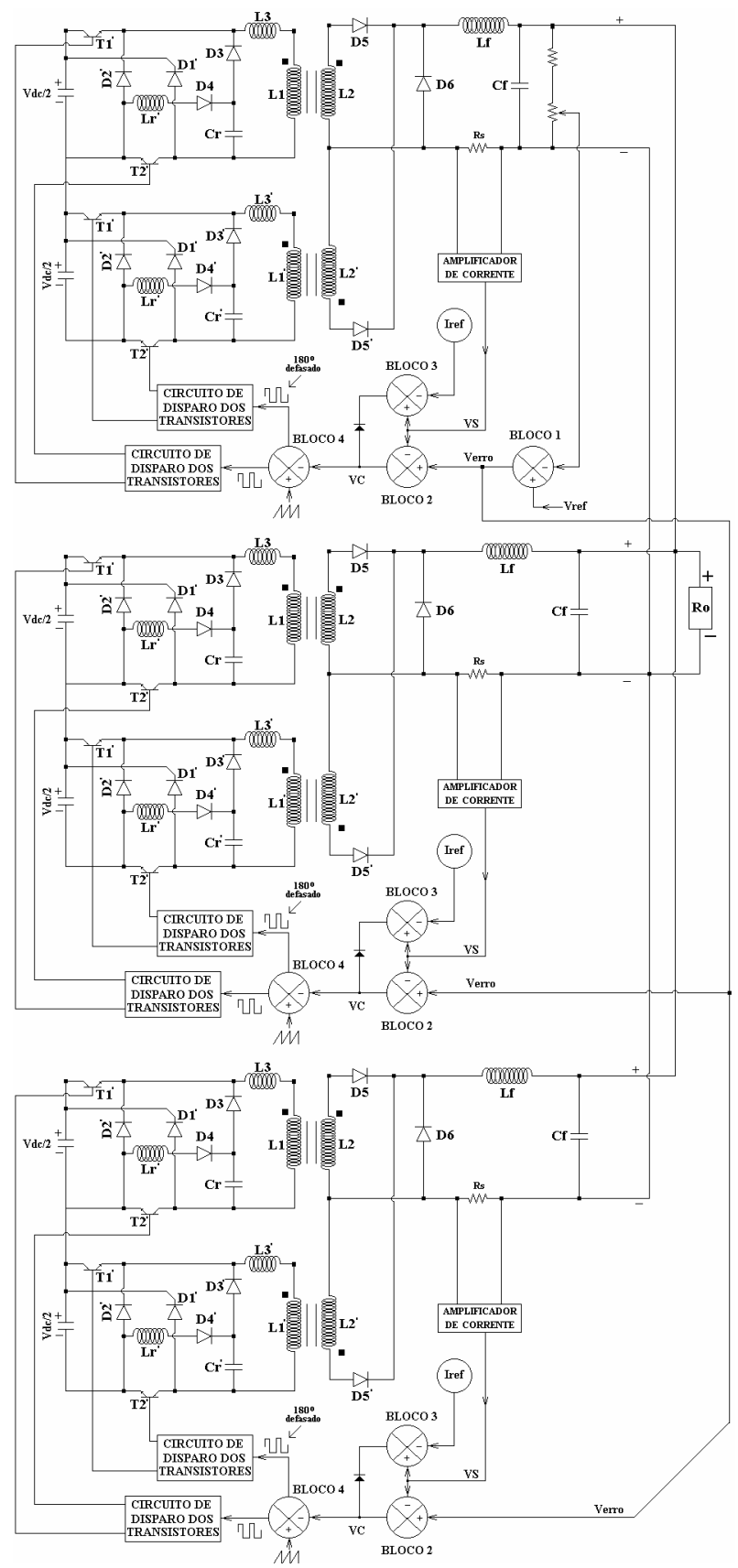

Figura 12 - Diagrama de Blocos Para o Controle da Fonte Trifásica.

Os terminais positivo e negativo de cada módulo estão conectados em paralelo, consequentemente, ao mesmo potencial. Considerando que os valores dos 
sensores de corrente são iguais, e implementando a realimentação da tensão de saída no módulo mestre, tem-se a tensão Verro e esta irá para os módulos escravos. Em cada módulo, o circuito responsável para a obtenção do sinal de tensão Vs possui o mesmo ganho, e irá acompanhar o sinal de Verro. O mesmo princípio é válido para Iref, ou seja, Iref é comum a todos os módulos.

\section{Exemplo de Projeto}

O projeto deve ser feito com os valores adequados de a (maior que 0,3 ) para que o conversor funcione na região PWM como mostrado na Figura 9. Uma outra condição a ser satisfeita é que a freqüência de ressonância seja pelo menos dez vezes maior que a frequiência de chaveamento. Dessa forma, pode-se calcular os valores dos elementos ressonantes que compõem a estrutura. As equações básicas para o cálculo do indutor e do capacitor de ressonância são aquelas dadas de (2) a (3). Os valores dos elementos ressonantes devem satisfazer os valores de $\alpha$ para que o conversor esteja na região de funcionamento PWM.

Dados do projeto:

Tensão de entrada $(\mathrm{Vdc})=300 \mathrm{~V}$;

Corrente de entrada $(\mathrm{Io})=20 \mathrm{~A}$;

Freqüência de Chaveamento $=100 \mathrm{kHz}$;

$\mathrm{Vo}=60 \mathrm{~V} ;$ Iout $=100 \mathrm{~A} ;$ Po $=6000 \mathrm{~W}$ e $\mathrm{a}=1$.

$\frac{f_{s}}{f_{o}}=0,1 \Rightarrow f o=1 \mathrm{MHz}$

$1 * 10^{6}=\frac{1}{2 \pi \sqrt{L r \cdot C r}} \Rightarrow C r=\frac{1}{4 * 10^{12} \pi^{2} \cdot L r}$

$\mathrm{a}=\frac{20}{300} \cdot \sqrt{\frac{L r}{C r}} \Rightarrow 1^{2}=\frac{400}{90000} \cdot L r^{2} \cdot 39,5 * 10^{12}$

$L r=2,39 \mu H \Rightarrow C r \cong 10,5 \eta F$
Pelo fato do valor de Cr não ser um valor comercial, adota-se um capacitor de $10 \mathrm{nF}$. Com isto, tem-se a $=1,03$, e o valor de $f_{o}=1,03 \mathrm{MHz}$.

\section{Técnicas de Supressão de RFI}

Os testes RFI possuem custos elevadíssimos, pois são realizados em laboratórios específicos. Sendo assim, antes de realizar aos testes, deve-se ter certeza de que o equipamento desenvolvido esteja com níveis aceitáveis, para não ocorrer um dispêndio financeiro desnecessário. Com isso, adota-se métodos práticos e eficientes para verificar se o equipamento sob teste tem baixo RFI. Para isto, basta ligar o equipamento próximo a equipamentos que funcionam com freqüências diversas, tais como televisores, rádios AM e FM. A verificação será visual e/ou auditiva. No caso da TV, aparecerão faixas, indicando que o equipamento precisa de filtros. Quanto ao Rádio, acorrerão "assovios" (TREVISO, 1999; CRUZ, 1996). Foram adotadas diversas técnicas para conseguir-se atenuações dos ruídos conduzidos e radiados, tais como: cuidado especial com o "layout", separação entre primário e secundário, utilização dos capacitores tipo X e Y para supressão dos ruídos modo comum e diferencial, blindagens nos transformadores e indutores, desacoplamento dos transformadores e indutores, blindagem do módulo, filtro de entrada blindado e "snubber" dissipativo em todos os semicondutores de potência.

A Figura 13 mostra o circuito elétrico projetado para um módulo, adotando todos a técnicas para a reduções dos ruídos RFI. 


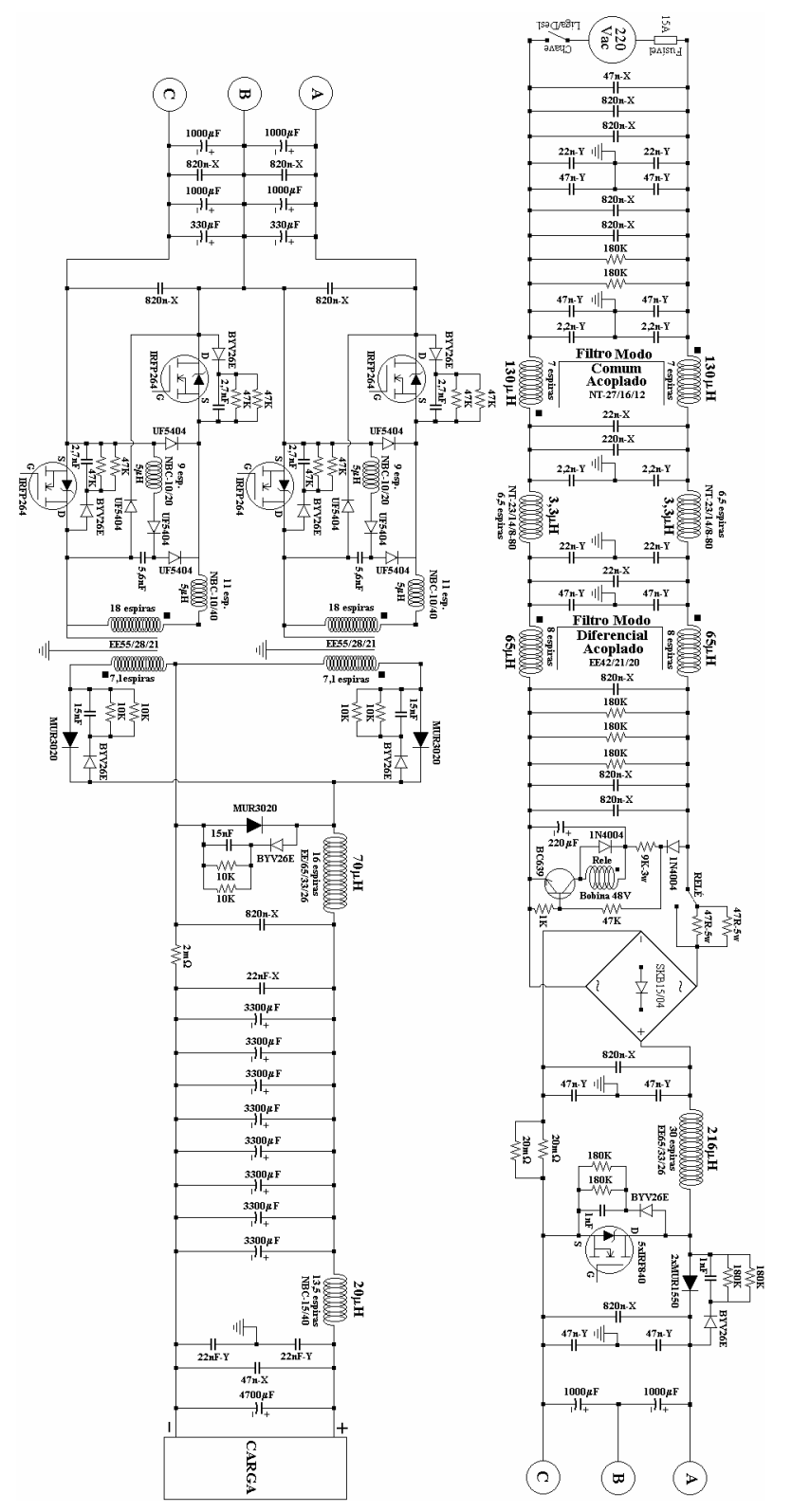

Figura 13 - Esquema Elétrico da Potência de Cada Módulo.

Ao ligar a fonte trifásica próximo a TV, observaram-se faixas transversais praticamente imperceptíveis, quanto ao rádio, não se observou qualquer alteração no som, indicando que a fonte possui baixo RFI.

\section{Resultados Experimentais}

Os resultados experimentais são obtidos do módulo mestre, pois a técnica de controle empregada, faz com que os demais módulos tenham o mesmo comportamento e a mesma resposta dinâmica da fonte trifásica. Além disso, o laboratório possui somente um Wattímetro “..TRUE RMS...”, o que reforçou a decisão de obter-se os resultados somente do módulo mestre (medidas mais confiáveis).

Na Figura 14 tem-se a forma de onda da tensão e corrente de entrada para a potência de 2000 Watts de saída.

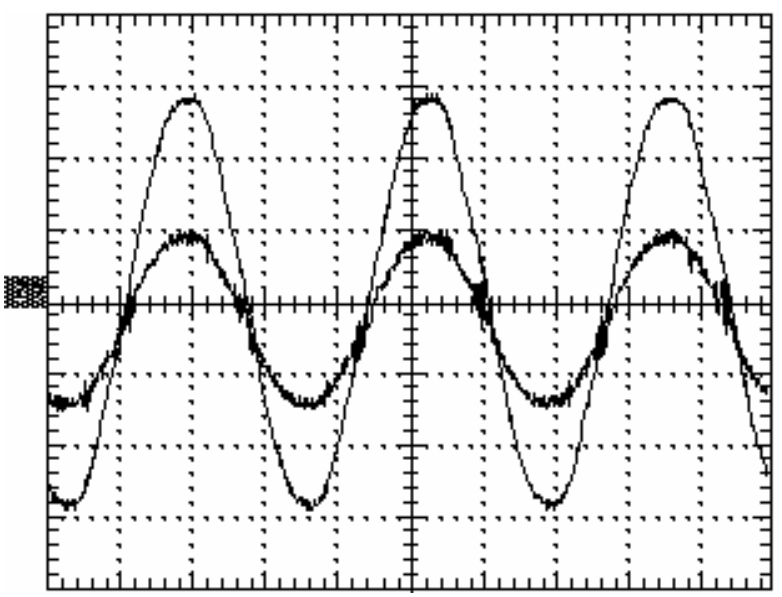

Figura 14 - Formas de Onda da Tensão e Corrente de Entrada Para Potência de 2000W de Saída.

A Figura 15 mostra a curva do fator de potência para potências variando de $300 \mathrm{~W}$ a $2000 \mathrm{~W}$ de saída.

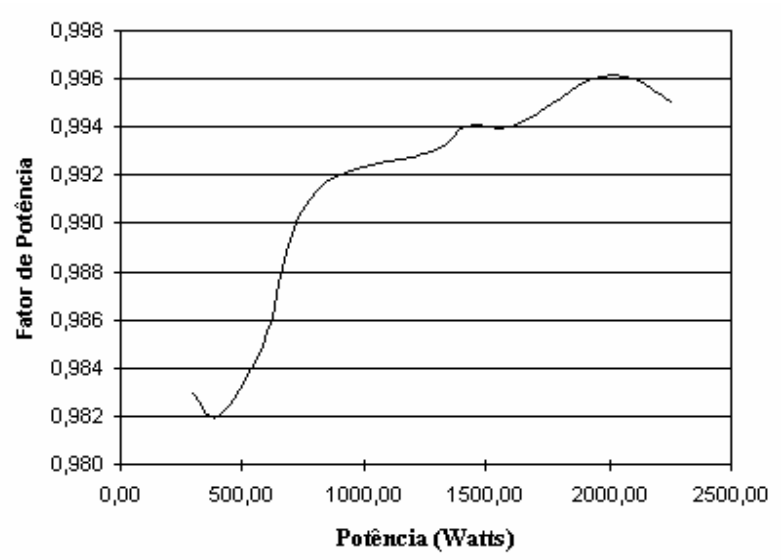

Figura 15 - Curva do Fator de Potência. 
A Figura 16 traz a forma de onda da tensão e corrente no mosfet que faz parte do circuito ressonante do conversor Forward a 2 transistores com "snubber" não dissipativo.

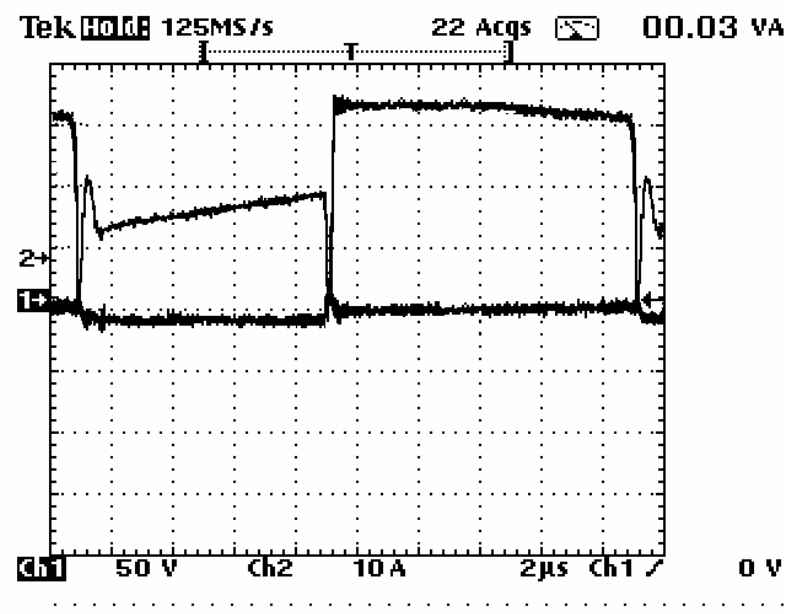

Figura 16 - Forma de Onda da Tensão e Corrente no Mosfet do Circuito Ressonante.

A Figura 17 traz o rendimento de cada módulo.

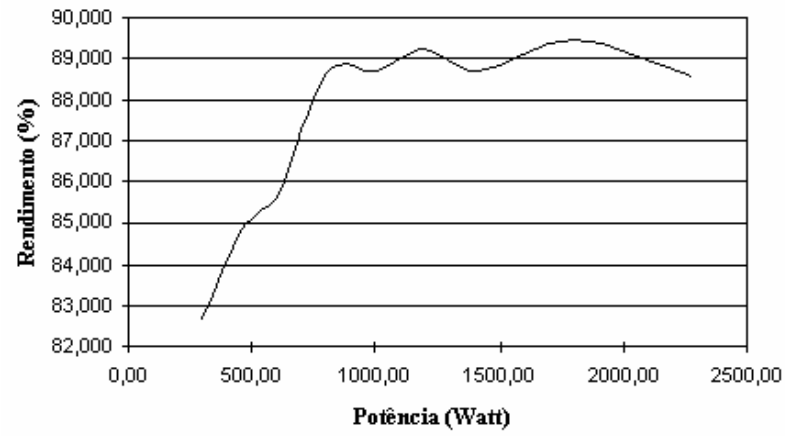

Figura 17 - Rendimento Global do Módulo Monofásico.

A Figura 18 expõe a resposta dinâmica para a fonte trifásica, com variação de carga de 50\% a 100\% e frequiência de $1 \mathrm{KHz}$.

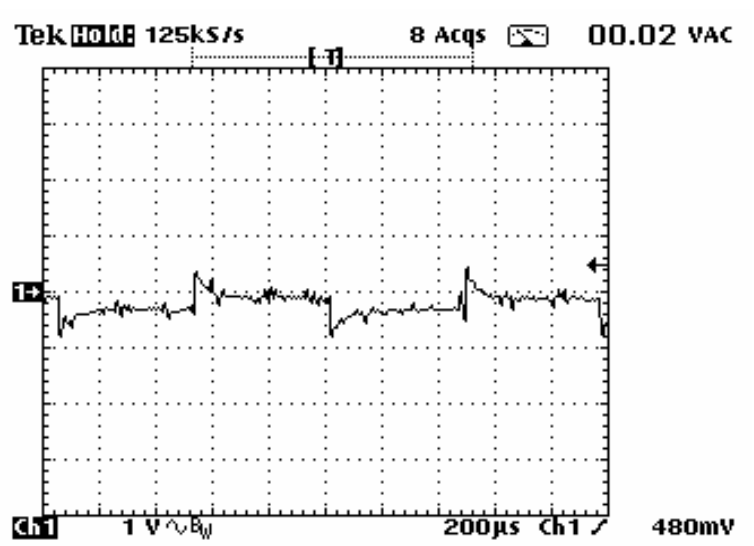

Figura 18 - Resposta Dinâmica da Fonte Trifásica Com Carga Variando de 3000W a 6000W.

Observa-se que o controle possui ótimo desempenho, pois não foram observadas oscilações no momento da variação de carga.

A Figura 19 mostra a fotografia da fonte trifásica.

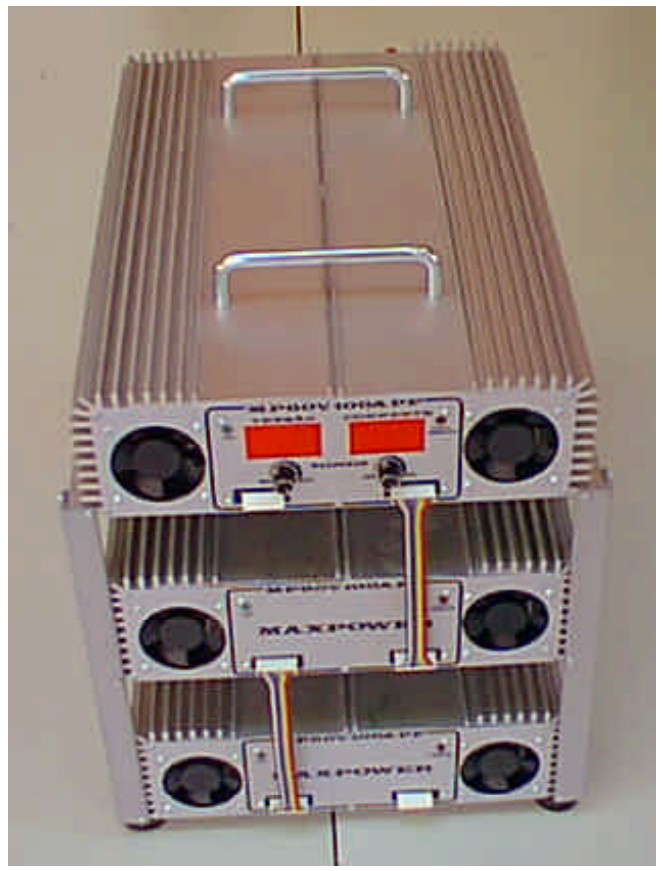

Figura 19 - Fotografia da Fonte Trifásica. 


\section{Conclusão}

Este artigo teve como objetivo, apresentar um retificador trifásico com fator de potência unitário, baixa distorção harmônica de corrente, reduzidos níveis de ruídos radiado e conduzido, alto rendimento, tensão de saída com isolação galvânica e potência de $6 \mathrm{KW}$ de saída $(60 \mathrm{~V}, 100 \mathrm{~A})$.

Isto tornou-se possível com a utilização do conversor Boost PWM convencional como préregulador e para a conversão $\mathrm{CC}-\mathrm{CC}$, dois conversores Forward a 2 transistores com "snubber" não dissipativo acoplados, ou seja, multi-nível.

O custo de material da fonte foi estimado em US\$ 1.326,13 dólares, cotados em São Paulo. Levando em consideração o lucro da empresas que varia de 40 a $50 \%$, o custo de material do equipamento ficaria bastante reduzido se for produzido em série e adquirindo-se os componentes diretamente dos fabricantes.

Os resultados experimentais comprovam a eficiência da fonte retificadora trifásica, com rendimento global de $89 \%$ e fator de potência de 0,996 para a potência nominal.

A estrutura da fonte (colocada em módulos para divisão de potência) possibilita especificar uma fonte para qualquer faixa de potência, não ficando limitada somente na estrutura trifásica.
Esta fonte pode ser utilizada em telecomunicações, laboratórios experimentais e até para soldagem de peças metálicas.

\section{Referências}

\section{ASSOCIAÇÃO BRASILEIRA DE NORNAS}

TÉCNICAS. NBR-12304: limites e métodos de medição de rádioperturbação em equipamentos para tecnologia da informação - (EIT). Rio de Janeiro, dez. 1991.

CRUZ, D. F. Contribuição ao estudo dos fenômenos de radio-interferência provocados por fontes chaveadas: análise, propostas de solução e resultados experimentais. 1996. Dissertação (Mestrado) Universidade Federal de Uberlândia, Uberlândia.

INTERNATIONAL ELECTROTHECNICAL COMMISSION. CISPR11: Limits and methods of measurement of electromagnetic disturbance characteristics of industrial, scientific and medical (ism) radio-frequency equipment. $2^{\text {th }}$ ed. Geneva, 1990.

TREVISO, C. H. G. Retificador de $6 \mathrm{KW}$, fator de potência unitário, trifásico, comutação não dissipativa na conversão cc/cc e controle sincronizado em freqüência. 1999. Tese (Doutorado) - Universidade Federal de Uberlândia, Uberlândia.

TREVISO, C.H.G. et al. A 1,5 KW two transistors forward converter using non-dissipative snubber. In: IEEE INTERNATIONAL SYMPOSIUM, 1998, Monterey. Proceeding... Monterey: IEEE, 1998. p.470-473.

UNITRODE. High power factor pregulator. Watertown, 1999. 